\title{
Pediatric isolated thoracic and/or lumbar transverse and spinous process fractures
}

\author{
Babatunde J. Akinpelu, MD, ${ }^{1,2}$ Scott L. Zuckerman, MD, ${ }^{3}$ Stephen R. Gannon, BS, ${ }^{3}$ \\ Ashly Westrick, MPH, ${ }^{3}$ Chevis Shannon, MBA, DrPH, ${ }^{3}$ and Robert P. Naftel, MD ${ }^{3}$
}

\begin{abstract}
${ }^{1}$ Department of General Surgery, Vanderbilt University; ${ }^{2}$ Department of Medicine, Meharry Medical College; and ${ }^{3}$ Department of Neurological Surgery, Vanderbilt University, Nashville, Tennessee
\end{abstract}

\begin{abstract}
OBJECTIVE Isolated transverse and spinous process fractures (TPFx and SPFx) in the thoracic and/or lumbar region have been deemed clinically insignificant in the adult population. This same rule is often applied to the pediatric population; however, little evidence exists in this younger group. The goal of this study was to describe the clinical, radiographic, and long-term data on isolated TPFx and SPFx in an exclusively pediatric population.
\end{abstract}

METHODS A retrospective chart review at Monroe Carell Jr. Children's Hospital at Vanderbilt University identified 82 pediatric patients with isolated TPFx and/or SPFx following a traumatic event between January 2000 and December 2013. Patient demographic information, presenting symptoms, radiographic characteristics, and follow-up data were collected. Follow-up was used to determine the outcome (presence of neurological deficits) of such injuries via complete physical examination and, when available, radiographic evidence.

RESULTS In the 82 identified patients, the mean age was $15.5 \pm 3.1$ years (mean is expressed \pm SD throughout), with 72 injuries (87.8\%) resulting from a motor vehicle, motorcycle, or all-terrain vehicle accident. There was a mean of $1.7 \pm$ 1.0 fractured vertebral levels involved and a mean of $1.8 \pm 1.1$ fractures was identified per patient. Seventy-one patients $(86.6 \%)$ needed bedside pain control, 7 (8.5\%) were prescribed a brace, and 4 patients $(4.9 \%)$ received a collar. Physical therapy was recommended for 12 patients (14.6\%). A total of $84.1 \%$ had follow-up, and the mean length of follow-up was $19 \pm 37$ months. No patients had true neurological deficits at presentation or follow-up as a result of their isolated fractures, whereas $95.1 \%$ had other associated system injuries.

CONCLUSIONS These data shows that there is no appreciable long-term complication associated with isolated thoracic and/or lumbar TPFx and/or SPFx in an exclusively pediatric population. Because these fractures are, however, associated with high-energy blunt trauma, they often result in associated soft-tissue or other skeletal injury. All pediatric patients in the cohort benefited from conservative management and aggressive treatment of their comorbidities.

http://thejns.org/doi/abs/10.3171/2015.10.PEDS15377

KEY WORDS fractures; vertebra; spine trauma; pediatric; transverse process fracture; spinous process fracture

$\mathrm{F}$ RACTURES of the thoracic and/or lumbar spine can cause neurological injury and/or lead to gross instability. Spinal fractures can categorically be grouped as stable or unstable. Stable thoracic and lumbar fractures do not cause structural loss of integrity and require no surgical intervention. ${ }^{1}$ Conversely, unstable thoracic and/or lumbar fractures often disrupt 2 or more of the 3 columns of the spine, ${ }^{3}$ and patients are at risk for developing neurological injury and/or deformity when these injuries are mismanaged. ${ }^{8}$ Several scoring systems exist to differentiate stable from unstable thoracic and/or lumbar injuries, stratifying patients who need operative stabilization; the most useful of these are the Thoracolumbar Injury Classification and Severity Score and the AOSpine classifica-

ABBREVIATIONS ATV = all-terrain vehicle; MVC = motor vehicle collision; $\mathrm{PT}=$ physical therapy; $\mathrm{SCl}=$ spinal cord injury; $\mathrm{SPFx}=$ spinous process fractures; TPFx = transverse process fractures.

SUBMITTED June 18, 2015. ACCEPTED October 21, 2015

INCLUDE WHEN CITING Published online February 19, 2016; DOI: 10.3171/2015.10.PEDS15377. 
tion system. ${ }^{7,11}$ Although both the Thoracolumbar Injury Classification and Severity Score system and the Denis' 3-column classification are restricted to the thoracolumbar region (T10-L2), extrapolating their concepts helps us to define patients who are at an increased risk of spinal cord injury (SCI). Differentiating stable from unstable spine fractures is of utmost importance in the setting of a potential spinal column injury. Spinous process fractures (SPFx) and transverse process fractures (TPFx) are considered to be stable. The spinous process is directed posteriorly at the junction of the lamina. Transverse processes project laterally from the junction of the lamina and the pedicle. Both structures serve as attachment sites for nearby muscle; however, neither is responsible for load bearing. In adults, TPFx and/or SPFx are typically managed with pain medication, rest, and possibly a brace or corset for comfort. Earlier studies have specified that consultation of the spine service was not recommended for isolated TPFx, concluding that these injuries are benign when isolated..$^{1,5}$

Despite a robust literature on isolated TPFx and/or SPFx in adults, the pediatric literature is sparse. Additionally, it is unclear whether these fractures are associated with other organ system trauma. The goal of our manuscript was to describe the clinical, radiographic, and longterm data on isolated TPFx and SPFx in an exclusively pediatric population.

\section{Methods}

We retrospectively reviewed patients evaluated for spinal injuries between January 2000 and December 2013 at the Monroe Carell Jr. Children's Hospital at Vanderbilt University. In this study, we defined TPFx and SPFx as a breakage in continuity of the specified bony prominence as evidenced on radiographic imaging (radiographs, CT scans, or MR images). The fracture(s) can be either displaced or nondisplaced. Only isolated fractures were included, meaning there was no other concomitant vertebral column injury.

Using ICD-9 codes, 1266 pediatric patients less than 19 years of age were identified as having a spinal column and/or cord-related injury at Monroe Carell Jr. Children's Hospital (Fig. 1). Patients with injuries other than those of interest and/or patients with injuries outside the thoracic and lumbar region were excluded. Ninety-six patients with TPFx/SPFx were initially identified, but 14 of them were excluded due to concomitant spinal fractures, in addition to the confirmed TPFx (11 patients) and SPFx (3 patients). Thus, the final cohort included 82 patients with true isolated TPFx and/or SPFx. Because all patients in our cohort suffered blunt traumatic injuries, they all went through routine trauma screening that included $\mathrm{CT}$ and/or radiographic scans, which revealed their TPFx and/or SPFx.

Patient demographic, radiographic, and follow-up data were collected. The admitting spine service was defined as either the orthopedic or neurosurgical team. To define a patient as being neurologically intact, the results of the physical examination were carefully reviewed for any signs of motor weakness, sensory deficits, or other deficits that could be attributed to the injury. Follow-up clinical

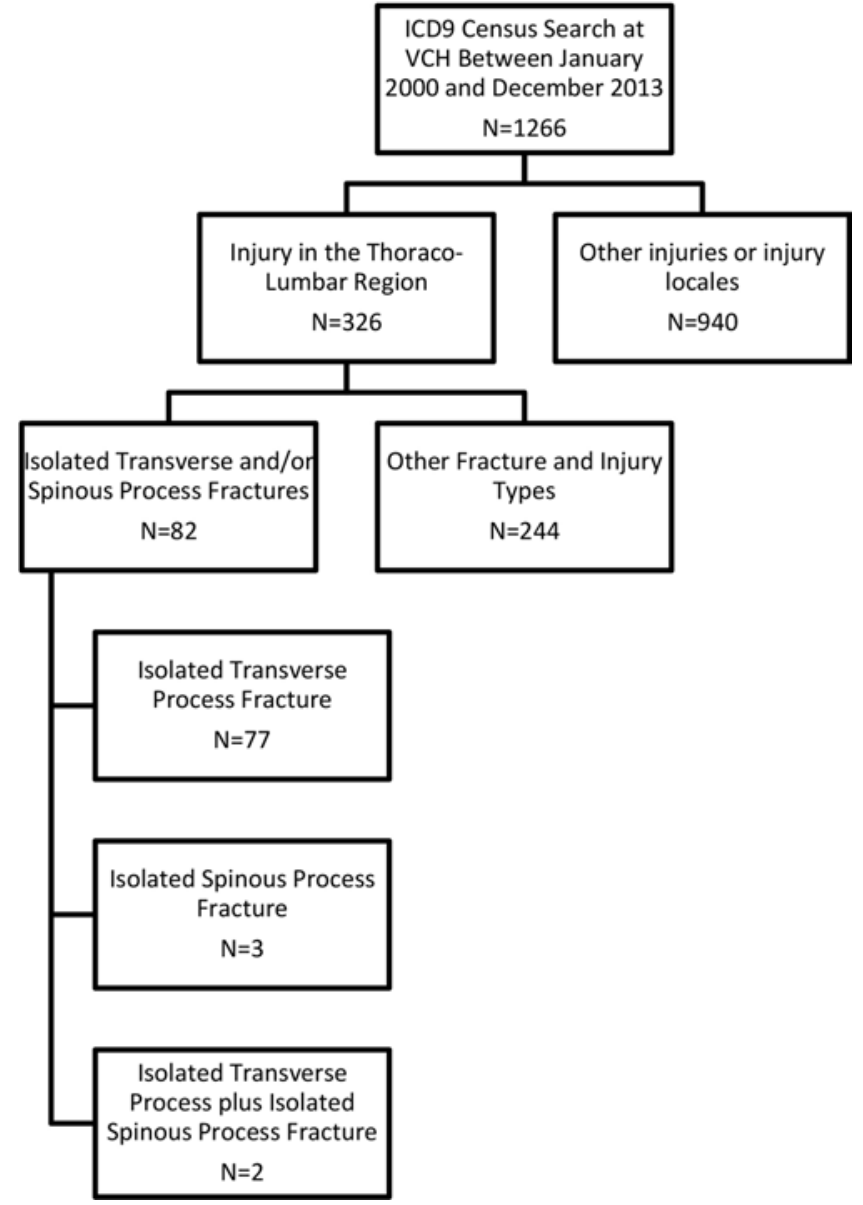

FIG. 1. Patient cohort flow chart-1266 pediatric patients were originally identified through an ICD-9 census; the cohort was then narrowed down to 82 patients identified as truly having an isolated TPFx or SPFx. VCH = Vanderbilt Children's Hospital.

encounters did not always include a radiographic study, but when it was appropriate, radiology report(s) from subsequent imaging were obtained. Since radiographic follow-up was only ordered when clinical evaluation demanded it, the imaging modality requested depended on the suspected medical condition being diagnosed or followed up on; as a result, the modality varied from patient to patient.

\section{Outcome Assessment}

Documented information included age, sex, race, admission/discharge dates, mechanism of injury, number of fractures observed, number of vertebral levels involved, whether there was evidence of SCI, whether spine service was consulted, treatment modality prescribed, length of clinical follow-up, presence of pain at follow-up, and associated injuries (skeletal or organ tissue damaged). The mechanism of injury was categorized into motor vehicle collision (MVC), motorcycle accident, all-terrain vehicle (ATV), fall, assault, sports-related, and pedestrian injury. Clinical follow-up was defined as the last clinical encoun- 
ter with the patient in which a comprehensive history and physical examination were obtained. Length of followup was defined as the difference between the date of last clinical follow-up with Vanderbilt and the patient's date of discharge.

Neurological deficits were assessed by reviewing the patient's physical examination (including motor and/or sensory deficits); therefore evidence of SCI was determined by clinical examination and not radiographically. In a few instances, an MRI study was performed due to conflicting findings on physical examination. These MR images were reviewed by radiologists and the treating spine team (orthopedic or neurological surgeons). Pain was assessed using a standardized method, based on a clinical intake form recorded during each follow-up visit. This form subjectively asked whether the patient was still having pain while describing its intensity (on a scale of 1-10) and location. The form also asked patients whether they could complete appropriate activities of daily living (and restrictions), along with miscellaneous questions such as educational needs, nutrition, and social environment.

\section{Results}

In the final cohort of 82 patients with isolated TPFx and SPFx, 52 (63.4\%) were male. This percentage was similar to the initial cohort (1266 patients) identified by ICD-9, where $61.9 \%$ (784) were male. Also, 72 (88\%) were categorized as Caucasian. The mean age was $15.5 \pm 3.1$ years, and patients were admitted for a mean of $4.2 \pm 4.8$ days (mean is expressed \pm SD throughout).

In terms of injury mechanism, $72(88 \%)$ were due to MVC, motorcycle, or ATV. There was a mean of $1.7 \pm$ 1.0 vertebral levels involved. Forty-seven (57.3\%) patients had single-level involvement, and all others had multilevel involvement (Table 1). The lumbar vertebrae were more frequently injured than the thoracic region, at a ratio of approximately 2:1. Overall, injuries at L-1 and T-1 were most prevalent in our cohort (Fig. 2). Isolated TPFx and SPFx in the thoracic and/or lumbar region were often associated with injuries to other organ systems, including chest (43.9\%), abdominal (37.8\%), and head injuries (37.8\%).

Computed tomography was used to confirm the fractures in 81 of the 82 patients, and the remaining patient had the fracture confirmed with radiography. An MRI study was additionally used to assess soft-tissue injury in 2 patients, one for suspicion of SCI and the other for suspicion of ligamentous damage. In both cases, further injury was ruled out.

The spine service was consulted in 34 of 82 (41.5\%) patients; a neurosurgery consult was requested for 14 (17.1\%) patients, whereas the orthopedic service was consulted in $20(24.4 \%)$ patients. Seventy-one $(86.6 \%)$ patients received analgesic medications for pain control and required no further treatment for the spinal fracture. A minority of patients underwent bracing. A cervicothoracic collar was used in 4 (4.9\%), and a thoracolumbar spinal orthosis was used in 7 (8.5\%). Physical therapy (PT) was recommended for $12(14.6 \%)$ patients to address pain complaints and assist with mobility. Of the 12 patients in whom PT
TABLE 1. Injury characteristics in 82 patients with isolated TPFx and/or SPFx

\begin{tabular}{|c|c|}
\hline Characteristic & No. of Patients (\%) \\
\hline \multicolumn{2}{|l|}{ Mechanism of injury } \\
\hline MVC & $51(62)$ \\
\hline ATV & $8(10)$ \\
\hline Pedestrian & $7(9)$ \\
\hline Fall & $7(9)$ \\
\hline Motorcycle collision & $6(7)$ \\
\hline Sports & $2(2)$ \\
\hline Assault & $1(1)$ \\
\hline \multicolumn{2}{|l|}{ No. of vertebral levels affected } \\
\hline 1 & $47(57)$ \\
\hline 2 & $20(24)$ \\
\hline 3 & $8(10)$ \\
\hline 4 & $6(7)$ \\
\hline 5 & $1(1)$ \\
\hline \multicolumn{2}{|c|}{ Associated injuries w/ isolated TPFx \& SPFx } \\
\hline Chest injury & $36(44)$ \\
\hline Abdominal injury & $31(38)$ \\
\hline Cranial & $31(38)$ \\
\hline Lower extremity & $19(23)$ \\
\hline Pelvic fracture & $9(11)$ \\
\hline Upper extremity & $9(11)$ \\
\hline \multicolumn{2}{|l|}{ Prescribed treatment } \\
\hline None & $71(87)$ \\
\hline Brace & $7(9)$ \\
\hline Collar & $4(5)$ \\
\hline Surgery & $0(0)$ \\
\hline
\end{tabular}

was prescribed, 10 had significant skeletal fractures (including 6 lower-extremity fractures, 2 pelvic fractures, 1 upper-extremity fracture, and 1 fractured clavicle), 1 had a pulmonary contusion, and 1 suffered extensive abdominal injuries.

Sixty-nine $(84.1 \%)$ patients had subsequent clinical follow-up, with a mean of $19 \pm 37$ months. Radiological follow-up was not common; only $18(26.1 \%)$ of the 69 patients underwent imaging at follow-up, including 15 radiographs and 3 CT scans ( 1 of the patients in whom radiographs were obtained also underwent MRI). On review of all follow-up imaging, there was no evidence of spinal instability, deformity, or treatment failure associated with the TPFx or SPFx.

All patients underwent a neurological examination on presentation and at follow-up. At presentation, $98.8 \%$ (81 of 82) were neurologically intact; 1 patient presented with paralysis in both legs but regained function bilaterally within 48 hours of admission-SCI was ruled out with extensive imaging. Additionally, 1 patient complained of paresthesias in the fingertips, but this was probably unrelated to the injury, a fracture was at the left L-4 transverse process. At follow-up, all 69 patients were neurologically 


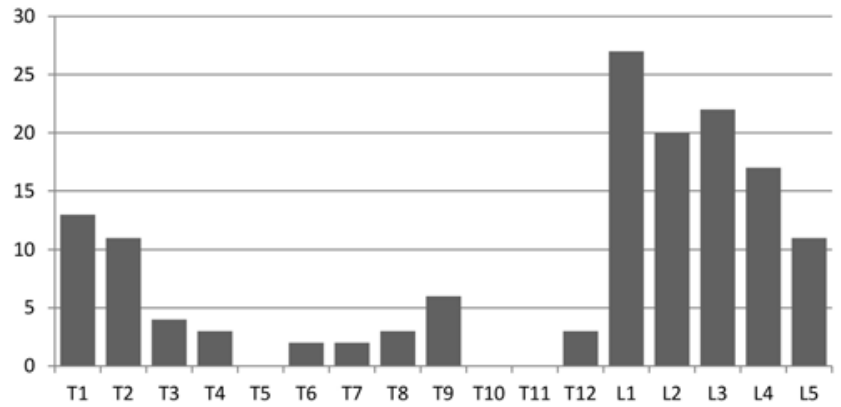

FIG. 2. Bar graph showing distribution of fractures per vertebral level; the $x$ axis denotes the vertebral level and the $y$ axis represents the number of fractures identified in the cohort at that particular level. The distribution reveals that isolated TPFx and/or SPFx have a higher likelihood of occurring in the lumbar versus the thoracic region.

intact, although 1 patient complained of lingering paresthesias in the fingertips that has improved since injury.

Patients who had isolated TPFx/SPFx in the thoracic region had a $70.0 \%(21 / 30)$ probability of having either a head $(50.0 \%)$ or chest injury $(50.0 \%)$, whereas the probability of having an abdominal injury was lower, at $20.0 \%$ (Table 2). On the other hand, patients who had these fractures in the lumbar region had a higher probability of abdominal injury (44.4\%), and a marginally lower probability of chest injury (40.7\%), but a lower probability of head injury $(29.6 \%)$ than that seen in patients with fractures in the thoracic region.

Eleven $(15.9 \%)$ of 69 patients noted residual pain during their first clinical encounter after hospitalization, with $3(4.3 \%)$ of them describing back pain. Of the 3 patients with back pain, 1 had low-back pain, 1 had paraspinal tenderness, and 1 had costovertebral angle tenderness. Of note, the patient who had the costovertebral angle tenderness suffered a renal laceration during the initial injury, and the patient who had paraspinal tenderness was only 2 weeks out from hospitalization. Data gathered for the last clinical encounter showed that only 7 (10.1\%) still had some form of pain, and this was primarily due to 6 of them not having longer-term follow up-the average time from last follow-up for these 6 patients was 17.2 days.

Only 1 patient who had a lengthy clinical encounter remained in pain - this patient initially had internal organ injuries (chest and abdomen), along with a pelvic fracture and bilateral femur fractures needing rods. Four years after the initial injury, the patient was again struck by an automobile and sent to the emergency room with scalp lacerations, abrasions and contusions, and a fractured right foot. The CT scans obtained during the subsequent hospitalization showed no injury to the spine or pelvis, but the patient's subjective pain got worse after this accident.

None of the 69 patients reported difficulty performing their activities of daily living. Our patients' pain was primarily managed pharmacologically. At discharge, 60 patients were prescribed narcotics (Percocet/Lortab/Roxicodone), 6 patients received only over the counter medications, and 3 received no pain medications.
TABLE 2. Injury characteristics per region of the vertebrae

\begin{tabular}{lrc}
\hline \multirow{2}{*}{ Characteristic } & \multicolumn{2}{c}{ No. w/ Injury $(\%)^{*}$} \\
\cline { 2 - 3 } & Thoracic & Lumbar \\
\hline No. of patients & 30 & 54 \\
\hline Associated injuries w/ isolated TPFx \& SPFx & & \\
\hline Chest injury & $15(50)$ & $22(41)$ \\
\hline Abdominal injury & $6(20)$ & $24(44)$ \\
\hline Cranial/head & $15(50)$ & $16(30)$ \\
\hline Lower extremity & $8(27)$ & $12(22)$ \\
\hline Pelvic fracture & $3(10)$ & $7(13)$ \\
\hline Upper extremity & $7(23)$ & $2(4)$ \\
\hline
\end{tabular}

* Two patients had spinal injuries in both the thoracic and lumbar region; hence the number adds up to 84 instead of 82 (the total number of patients).

\section{Discussion}

The goal of our study was to describe the clinical, radiographic, and long-term data on isolated TPFx and SPFx in an exclusively pediatric population. As expected, there was no association between isolated TPFx/SPFx and spinal column instability, spinal cord or nerve root injury, or neurological deficit. This lack of association has been noted in adults but never before in pediatric patients. ${ }^{1,5}$

Isolated TPFx and SPFx are common injuries. Patten et al. describe the incidence of TPFx in patients with abdominal trauma as $7.3 \%$ after screening with a helical abdominal CT. ${ }^{10}$ With the frequent incidence of these injuries, long-term clinical data are needed to determine optimal management. We saw no complications or deficits associated with isolated TPFx and SPFx. We also saw no evidence of sustained pain or diminished ability to perform normal activities of daily living within our cohort. The lack of association between these types of fractures and longer-term morbidity is important to know so that unnecessary studies, interventions, and bracing are avoided. Additionally, precautions such as bed rest and use of the log roll can be avoided because of the stability of these injuries.

Our cohort described 11 of 82 patients (13.4\%) in whom either a brace or collar was prescribed. All 4 patients treated with a cervical collar sustained injuries to their head (3 patients) or upper thoracic region (1 patient); hence a collar was used until the patient could be cleared weeks later. Seven patients with midthoracic to lumbar TPFx/SPFx were prescribed a thoracolumbar spinal orthosis. Due to the retrospective nature of this study, it is difficult to ascertain why exactly braces were prescribed, because there is no evidence that any brace improves prognosis or outcome in the setting of TPFx/SPFx. The most likely reason was pain management and comfort. According to our longerterm data, patients healed appropriately without braces or collars. However, this study does not provide conclusive evidence that these devices do not assist with the healing process.

Although isolated TPFx/SPFx do not cause neurological injury or spinal instability, they are often associated with solid organ injuries due to the high-energy blunt trauma. ${ }^{910,12}$ In our study, it was noted that these associated 
injuries varied per vertebral region. Chest injury was common with higher thoracic injuries, and abdominal trauma was seen with lower thoracic or lumbar fractures. Trauma teams should have a high index of suspicion for other organ system injuries in patients with isolated TPFx/SPFx.

In this study, the spine service was not consulted in $58.5 \%(48 / 82)$ of cases. Delving further into the data, we see that of the 20 orthopedic consults received, 14 (70.0\%) patients had significant skeletal fractures such as pelvic and upper- and/or lower-extremity fractures needing stabilization. Eleven (78.6\%) of the 14 neurosurgery consults were in patients with head and/or scalp injuries, whereas 1 patient presented with transient lower-extremity paralysis.

Although earlier studies discussed these injuries as posing only a minor risk of morbidity, ${ }^{2}$ our studies show vertebral level-specific associated soft-tissue and skeletal injuries that require prompt medical attention. One of the patients in our study suffered an injury extensive enough to involve 4 vertebrae in the lumbar region (7 isolated fractures), but suffered no long-term morbidity as a result. In fact, Han and Sohn discussed a case report of a patient who sustained 12 contiguous spinous process fractures from C-4 to T-8 during a motorcycle crash, with good clinical outcome, although the patient was 34 years old. ${ }^{4}$

Our cohort included 9 patients who were 10 years or younger, and their associated injury characteristics were similar to our older pediatric patients. Five of the younger patients had isolated TPFx within the thoracic region, whereas the remaining 4 had isolated TPFx located within the lumbar region. All 4 patients who had injuries to their lumbar vertebrae suffered abdominal injuries, including 1 who suffered a pelvic fracture. Three $(60.0 \%)$ of the younger patients who had injuries in their thoracic region also had injuries to their head-again proving region-specific associated injuries. Also, although the biomechanical characteristics of pediatric patients in our age group (15.5 \pm 3.1 years) as a whole are similar to those of a younger adult, our paper benefits from a more focused patient population that solely represents the pediatric group. Other studies that are not specific will include elderly patients; this will cause an inherent skew in the data, because some of those patients have osteoporosis or other ligamentous or tendon stiffness, which are less prevalent within the pediatric population. As with the adult population, pediatric patients will benefit from aggressive pain management and early mobilization, as tolerated. Early mobilization will help improve functionality while also reducing the risk of developing deep vein thrombosis, along with its sequelae.

Our study is not without limitations. One limitation was that we concluded the absence of neurological injury by the absence of deficits on physical examinationslargely due to the retrospective nature of the study. We believe that this is clinically sufficient because there were no long-term complications noted; however, muscle soreness or pain issues could have been missed. ${ }^{6}$ Also, it was difficult to determine how much of the PT was related to the isolated TPFx or SPFx, because our patients had comorbidities that would benefit from PT. Additionally, we had no way of confirming if PT was completed, nor could we quantify how much of its effect improved patient prognosis with regard to isolated TPFx or SPFx. The PT was often ordered because a significant portion of our patients incurred severe skeletal injuries (cranial, pelvic, and extremities) that were also managed by orthopedic and neurological surgeons.

Again, due to the retrospective nature of the study, we were only able to assess pain via a clinical intake form, and although the form was not extensive, we were able to show that our patient's pain eventually resolved over time. Our study would have benefited from regular, specific clinic intervals so that we could track trends in the amelioration of the pain, but our clinic return times were not standardized and longer-term follow-ups were at the patient's discretion. Despite these limitations, we believe that our data highlight that these types of fractures only pose a minor risk to the spinal column of the pediatric patient, as is also shown in the adult population.

\section{Conclusions}

In analyzing these single-institution pediatric data over a 14-year period, we found no long-term complications associated with isolated thoracic and/or lumbar TPFx and/ or SPFx. When imaging scans reveal an isolated TPFx or SPFx after a traumatic event, care should be taken to rule out associated soft-tissue or skeletal injuries. Pediatric patients will benefit from conservative management and aggressive treatment of their comorbidities.

\section{Acknowledgments}

We thank everyone who volunteered their tireless efforts to this project, especially Vanderbilt's Surgical Outcomes Center for Kids (SOCKs). Without SOCKs' guidance and generosity, this manuscript would not have been possible.

\section{References}

1. Bradley LH, Paullus WC, Howe J, Litofsky NS: Isolated transverse process fractures: spine service management not needed. J Trauma 65:832-836, 2008

2. Daniels AH, Sobel AD, Eberson CP: Pediatric thoracolumbar spine trauma. J Am Acad Orthop Surg 21:707-716, 2013

3. Denis F: The three column spine and its significance in the classification of acute thoracolumbar spinal injuries. Spine (Phila Pa 1976) 8:817-831, 1983

4. Han SR, Sohn MJ: Twelve contiguous spinous process fracture of cervico-thoracic spine. Korean J Spine 11:212-213, 2014

5. Homnick A, Lavery R, Nicastro O, Livingston DH, Hauser CJ: Isolated thoracolumbar transverse process fractures: call physical therapy, not spine. J Trauma 63:1292-1295, 2007

6. Inaba K, DuBose JJ, Barmparas G, Barbarino R, Reddy S, Talving P, et al: Clinical examination is insufficient to rule out thoracolumbar spine injuries. J Trauma 70:174-179, 2011

7. Lee JY, Vaccaro AR, Lim MR, Öner FC, Hulbert RJ, Hedlund $\mathrm{R}$, et al: Thoracolumbar injury classification and severity score: a new paradigm for the treatment of thoracolumbar spine trauma. J Orthop Sci 10:671-675, 2005

8. Louis R: Spinal stability as defined by the three-column spine concept. Anat Clin 7:33-42, 1985 
9. Miller CD, Blyth P, Civil ID: Lumbar transverse process fractures - a sentinel marker of abdominal organ injuries. Injury 31:773-776, 2000

10. Patten RM, Gunberg SR, Brandenburger DK: Frequency and importance of transverse process fractures in the lumbar vertebrae at helical abdominal CT in patients with trauma. Radiology 215:831-834, 2000

11. Vaccaro AR, Oner C, Kepler CK, Dvorak M, Schnake K, Bellabarba C, et al: AOSpine thoracolumbar spine injury classification system: fracture description, neurological status, and key modifiers. Spine (Phila Pa 1976) 38:2028-2037, 2013

12. Xia T, Tian JW, Dong SH, Wang L, Zhao QH: Non-spinalassociated injuries with lumbar transverse process fractures: influence of segments, amount, and concomitant vertebral fractures. J Trauma Acute Care Surg 74:1108-1111, 2013

\section{Disclosures}

The authors report no conflict of interest concerning the materi- als or methods used in this study or the findings specified in this paper.

\section{Author Contributions}

Conception and design: Akinpelu, Westrick, Shannon, Naftel. Acquisition of data: Akinpelu, Westrick, Shannon. Analysis and interpretation of data: Akinpelu, Zuckerman. Drafting the article: Akinpelu, Zuckerman, Gannon. Critically revising the article: Akinpelu, Zuckerman, Gannon, Naftel. Reviewed submitted version of manuscript: Akinpelu, Zuckerman, Gannon, Westrick, Naftel. Approved the final version of the manuscript on behalf of all authors: Akinpelu. Study supervision: Zuckerman, Shannon, Naftel.

\section{Correspondence}

Babatunde J. Akinpelu, Department of General Surgery, Vanderbilt University Medical Center, 116121 st Ave. S, D-4309 Medical Ctr. N., Nashville, TN 37232. email: babatunde. akinpelu@vanderbilt.edu. 